\title{
Dietitians Meet the NHS Agenda for Improved Patient Care and Clinical Outcomes through Nutrition Support Study as a Module Masters Level - A Preliminary Report
}

\author{
Fiona McCullough*, Ann Micklewright \\ Division of Nutritional Sciences, School of Biosciences, University of Nottingham, UK
}

Received: October 24, 2013; Accepted: January 20, 2014; Published: January 25, 2014

*Corresponding author: Fiona McCullough, Division of Nutritional Sciences, School of Biosciences, University of Nottingham, LE12 5RD, UK, Tel: 0115951 6118; Fax: +44 (0)115 951 6122; E-mail: Fiona.mccullough@nottingham.ac.uk

\begin{abstract}
Aim: The aim of the project was to evaluate the impact of a Master's module in nutrition support to provide dietetic managers with evidence of changed practice from their staff including improved knowledge, confidence and change in practice including patient outcomes as part of a continuing education approach.

Background: From 2008/09 the East Midlands Strategic Health Authority provided LBR (Learning beyond registration) funding for a Masters module in Nutrition Support for graduate dietitians with 1-4 years experience working in its catchment area (known as band 5 and 6). The module comprised elements of pre-course work, taught and interactive peer working sets, a work place audit and a written case study.
\end{abstract}

Method: Delegates from the first two modules were invited by means of an electronic questionnaire (covering a range of questions including about the content that had been learnt and the resulting effect on patient care) to evaluate the course and to see whether it had had any effect on practice.

Results: Feedback was very positive in that delegates felt more confident that they were working with a strong evidenced based knowledge which made them more pro-active in interpreting biochemistry and recommending appropriate interventions for complex cases. This, in addition to completion of a small audit project changed practice and improved patient care. Delegates self assessed themselves against nine clinical activities, two thirds of which were rated $>7 / 10$. Ratings for the lowest third were: nutrition diagnosis (4.2), objective setting (6.4), critically analysis of the working practice (6.7).

Conclusion: The participants felt, based on personal reflection, that the module had changed clinical practice and equipped delegates to take on more specialist roles in the future. However, both the module and this evaluation highlighted shortfalls in relation to clinical objective setting and measuring outcomes. The aim of the present project was to evaluate the impact of the module and provide dietetic managers with evidence (based on self-assessment) of changed practice from their staff including improved knowledge, confidence and change in practice including patient outcomes.

Keywords: Nutrition support; Enteral tube feeding; Parenteral nutrition (PN); Objectives; Outcomes; Disease related malnutrition (DRM)

\section{Introduction}

The aim of the SHA (Strategic Health Authority) funded LBR (Learning Beyond Registration) programme was and remains, to enhance clinical competency in such a way as to increase the quality of patient care, reduce clinical risk and improve patient outcomes.

In terms of evidencing the effectiveness of practice, outcome driven measures have been identified as the way forward to achieving the NHS ambition of a world class health services 'focused on outcomes and the quality standards that deliver them' [1].

The Nutrition Support (NS) module aimed to consolidate and expand upon the knowledge base of individuals already working in the field of nutrition support. This 20 credit MSc module was aimed at experienced band 5 and band 6 dietitians. It examined nutrition support strategies and provided dietitians with the skills and knowledge to assess patients' need's, design and monitor enteral tube feeding (feeding through a tube inserted via the nose into the stomach or jejunum, or inserted directly into the stomach or jejunum by endoscopy or surgery) and parenteral (feeding into a vein) nutrition regimens both on the general wards and in specialist areas such as critical care, renal disease and in straightforward children's conditions. The latter 2 conditions were included at the request of dietetic managers to enable participants to develop a level of confidence and competency in these areas as an initial step to becoming specialist dietitians.

Learning Outcomes covered knowledge and understanding, intellectual skills, and practical skills included to:

- Demonstrate an in depth knowledge and understanding of the theoretical principles of parenteral and enteral nutrition in the prevention and treatment of disease related malnutrition.

- Exercise evidence based clinical reasoning to critically evaluate specific practices relating to all aspects of enteral and parenteral nutrition. 
- Collect and integrate several lines of evidence and apply them in a balanced and flexible way including audit in clinical practice.

- Identify, communicate and minimise clinical risk.

The module aimed to develop a nutrition care process mindset, using the evidence based ADA (American Dietetic Association) Care Process [2] This promotes a stepped pathway approach that enables practitioners to set clear treatment objectives and measurable outcomes for individual patients important skills transferable across all areas of dietetic practice. In order to identify previous knowledge, pre-coursework was set (and assessed) which included critically appraising recent key publications in relation to the cost and clinical effectiveness of nutrition support $[3,4,5]$. Other assessed work included a parenteral nutrition case study and a small audit project identified from within each dietitian's own clinical practice.

The aim of the present project was to evaluate the impact of the module and provide dietetic managers with evidence of changed practice from their staff including improved knowledge, confidence and change in practice including patient outcomes.

\section{Method}

A questionnaire was constructed using the electronic Survey Monkey software, and was distributed by email to 20 participants who had undertaken the NS module over the previous 2 academic years. A copy of the questionnaire is included at the end of the paper.

\section{Key Results}

\section{Participants}

13 dietitians responded of whom, 11 were from the 2009/10 course and only 2 from the year before. Participants were experienced band 5 and new band 6 dietitians all with substantial experience of oral and enteral tube feeding (ETF) (but limited or no experience of parenteral nutrition (PN). Participant expectations of the course were generally positive.

\section{Response to questions}

1. Was a Masters module more appropriate to you than study days? Would you undertake another Masters level module?

$12(93 \%)$ respondents agreed that the MSC module was more appropriate than study days for a variety of reasons: already studying other modules towards a full masters degree or was hoping to do so; a masters module is a national recognised qualification and useful for CPD and career purposes; the module and assessed course work enabled participants to put what they had learned into practice in between module days; it was more informative, in depth and interactive than study days.

$1(7 \%)$ disagreed as she was not studying for a masters

Consequently, the same respondents were positive about undertaking another module for reasons similar to those expressed above.

\section{Please outline briefly how you expected your clinical practice to change when you enrolled for the module?}

All 13 participants answered the question and expectations were generally positive. Expectations were on the whole about increasing participants' knowledge and skills particularly in relation to PN, biochemistry and audit. Whilst this might not immediately change current practice it was preparation for the future, improving promotion prospects and enabling participants to cover for colleagues in specialist areas ('I was aware that I may be asked to cover maternity leave in the near future (before starting the course) which would involve covering PN and more of critical care'

One participant reported that "the knowledge and skilled gained would make me more confident to join in discussions with specialist colleagues about complex cases which in turn will increase my knowledge further."

There was also an expectation that the module would provide up to date evidence based clinical information, increase verbal and written communication skills, promote critical thinking and improve research and audit skills.

Networking with colleagues from other organisations was seen as an advantage, providing an opportunity to compare practice and learn from each other.

3. Do you feel that as a result you have been able to improve patient outcomes? know'

Over two thirds (9) respondents said 'Yes' whilst 4 'did not

Respondents stated that they had greater confidence that their knowledge was evidence based resulting in a more proactive approach to nutrition interventions. In particular, more involvement in interpreting clinical biochemistry results and in recommending and delivering appropriate and timely interventions were highlighted (and such decisions should be included in each dietitian's regular ongoing clinical supervision process). Two respondents said that their audit projects had identified areas of poor practice which had now been rectified to improve patient care.

\section{Setting treatment objectives}

$11(85 \%)$ respondents answered all 3 questions which are shown in figure 1 below along with the number of responses to each question. 55\% routinely set treatment outcomes, whilst $64 \%$ found SMART objectives difficult to write and would like further training.

There were 2 written responses: one respondent felt that objective setting was done routinely but not as the ADA Care Process which was taught on the course suggested and was not something she would take back to use in her Department. The other respondent said that she had done a lot about objective setting in her undergraduate course and it did not warrant the time spent on it in the MSC module. 


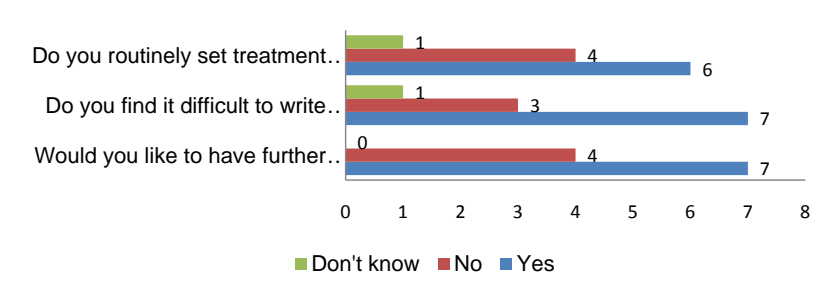

Figure 1: Response to questions about treatment objectives.

\section{Do you ROUTINELY measure clinical outcomes?}

Again only 11 respondents answered of whom 7 (64\%) said they routinely measured clinical outcomes, 2 did not and 2 did not know. 5 respondents commented that: outcomes were routinely measured through team audits (1); only simple measures such as weight in relation to intervention, lack of symptoms, height (2); through report back to senior staff (1); prevented through time constraints in clinical practice (1).

\section{Have you had an opportunity to use the renal/} paediatric information?

Of the 11 who answered the question 6 (55\%) had had the opportunity to work in one/both of these areas. 2 respondents were already working in renal dietetics and did not find the renal session useful. 4 others found the renal information transferable to other areas e.g. critical care. Only 1 had used the paediatric information.

\section{Do you now feel more confident to cover for a} specialist dietitian in renal or paediatrics?

Only 6/11 (55\%) said that they would feel confident in covering for a specialist renal or paediatric dietitian> others said they had not had the opportunity to undertake these roles, particularly paediatrics and would use the information as a resource if needed in the future.

\section{On a scale of 1 (low) to ten (high) please answer the following questions (Table 1)}

Respondents rated 9 questions relating to their own clinical practice. Average rating scores were calculated for each question. $6(66 \%)$ questions were rated $>7$ (range 7.5 to 8.8). The 3 lowest ranged from 6.7 to 4.2 : making a nutritional diagnosis (4.2), structuring work and setting objectives (6.4) and critically appraising practice and setting audit

\section{Discussion}

It is estimated that more than 3 million people in the UK suffer from Disease Related Malnutrition (DRM), the overall cost to health services is in the region of $€ 13$ billion pounds per annum. For malnourished patients there is an associated increased risk of morbidity and mortality [5]. In recent years a mammoth effort has been undertaken by BAPEN (British Society for Enteral \& Parenteral Nutrition) to introduce nutrition screening into hospital and the community [4]. If used correctly this allows timely identification of patients at risk or suffering from DRM and facilitates appropriate nutrition support interventions which improve patient outcomes and reduce healthcare costs [8].

Providing nutrition support to patients is a large part of the work of most dietitians, both in the community and in hospital. Whilst many have experience of supporting patients with oral and enteral tube feeding modalities, those at Band $5 \& 6$ are less versed in the use of parenteral nutrition (PN). When asked about their expectations of the course, increasing their knowledge and skills in relation to PN was a prominent theme. To this end a whole day was dedicated specifically to the management of PN and delegates were given time to work through a number of complex cases in peer learning sets. In addition a 3000 word PN case study was set as course work.

The pre-course work was set with the intention of ensuring that delegates became familiar with key publications which impacted on nutrition support and to critically appraise their organisations performance against the standards expected from these documents. Each participant then developed an audit idea through discussion with module tutors, dietetic line managers and their Trust audit teams. This resulted in a small project being implemented and presented. Participants highlighted strongly that these audit made improvements to patient care.

During the course, it became apparent whist discussing the ADA care process that participants were not routinely writing comprehensive intervention objectives. ('we know what we are doing in our head'). Just over half said that they routinely set objectives and more than two-thirds had difficulty writing SMART [6] objectives. This was evidenced throughout the course work with the majority failing to write clear objectives in their case studies and/or audit projects. Two thirds of respondents said they would like further training in this area.

Clinical outcomes were measured by around two thirds of respondents though these tended to be very simple measures such as weight in relation to dietetic treatment. According to the British Dietetic Association there are 'currently no UK validated sensitive and reliable dietetic outcome measures available for routine use' [7]. In practice, focus seems to be around dietetic management rather than outcome measures to provide evidence of efficacy of interventions [8].

The survey was sent by email to two cohorts from two consecutive years. The majority of respondents were from the second year. Dietitians at grade 5 and 6 tend to move jobs frequently so delegates may have been lost to contact. E-mail surveys are relatively new and the authors' limited experience with such surveys, suggest a poorer response than with postal surveys. However those who responded were positive about the course content with its mixture of taught, interactive, pre and post course activities. All but one said they preferred to undertake a Master's module rather than separate study days and would take a further module if appropriate to their clinical practice

In terms of the self assessment (table 1), delegates rated themselves 7 and above on all except 3 criteria: Nutrition 
Table 1: Respondents ( $\mathrm{n}=11)$ ratings for questions relating to clinical practice.

\begin{tabular}{|l|l|l|l|l|l|l|l|l|l|l|l|}
\hline Answer Options & $\mathbf{1}$ & $\mathbf{2}$ & $\mathbf{3}$ & $\mathbf{4}$ & $\mathbf{5}$ & $\mathbf{6}$ & $\mathbf{7}$ & $\mathbf{8}$ & $\mathbf{9}$ & $\mathbf{1 0}$ & Av. Rating \\
\hline My work is now structured and I set objectives & 0 & 0 & 1 & 0 & 3 & 0 & 4 & 3 & 0 & 0 & 6.4 \\
\hline I frequently make a nutritional diagnosis & 2 & 1 & 2 & 1 & 1 & 2 & 1 & 1 & 0 & 0 & 4.2 \\
\hline I now understand clinical biochemistry better & 0 & 0 & 0 & 0 & 0 & 0 & 4 & 4 & 3 & 0 & 7.9 \\
\hline I look for audit dates and opportunities & 0 & 0 & 0 & 0 & 0 & 1 & 4 & 5 & 0 & 1 & 7.6 \\
\hline $\begin{array}{l}\text { I critically analyse practice in my own organisation and } \\
\text { identify shortcomings in clinical practice and set audit }\end{array}$ & 0 & 0 & 0 & 0 & 2 & 2 & 4 & 3 & 0 & 0 & 6.7 \\
\hline I feel more confident in the area of ethical decision making & 0 & 0 & 0 & 1 & 0 & 1 & 2 & 6 & 0 & 1 \\
\hline I take the patients expressed needs into account more & 0 & 0 & 0 & 0 & 1 & 0 & 3 & 5 & 1 & 1 & 7.5 \\
\hline I enable the patient to be part of the decision making process & 0 & 0 & 0 & 0 & 0 & 0 & 1 & 3 & 5 & 2 \\
\hline I feel that I am becoming an independent competent practitioner & 0 & 0 & 0 & 0 & 0 & 0 & 0 & 5 & 3 & 3 & 8.7 \\
\hline
\end{tabular}

Diagnosis was introduced as part of the ADA care process, and as such is a new concept. This will be difficult to put into operation by individuals unless their whole department is convinced of its value; objective setting and developing audits also scored below 7. In hindsight, these question should have been included with the pre-course work to enable a before and after comparison to be made.

\section{Conclusion}

The current preliminary report suggests that Dietitians have been supported to provide improved patient care and clinical outcomes through Continuing Professional Development at Masters Level. Of particular note were the majority of respondents who said that they now had greater competency because their knowledge was more evidenced based. This enabled them to take a more pro-active approach, particularly in interpreting biochemical data and recommending appropriate and timely nutrition interventions and thus improve patient care.

However, the course work and this evaluation identified the need for dietitians to develop outcome measures. Nutrition support interventions have the potential to make a difference to both clinical and financial outcomes. More than ever, in this current economic climate, it is essential that outcome measures are produced to evidence the value of timely and appropriate nutrition support interventions.

\section{References}

1. DOH White Paper (2010) Equity and excellence: Liberating the NHS.

2. Lacey C, Cross N (2002) A problem based nutrition care model that is diagnostic driven and allows for monitoring and managing outcomes. J Am Diet Assoc 102 (4): 578-588.

3. NICE clinical Guideline (2006) Nutrition support for adults: Oral nutrition support, enteral tube feeding and parenteral nutrition.

4. BAPEN (2005) Malnutrition Universal Screening Tool 'MUST'.

5. Elia M, Stratton RJ, Russell C, Green C, Pang, F., et al. (2010) Health Economic Report on Malnutrition in the UK. The cost of diseaserelated malnutrition in the UK \& economic considerations for the use of oral nutritional supplements (ONS) in adults.

6. BDA (2010) Proposed model for dietetic outcomes. British Dietetic Association, Birmingham, England.

7. Weekes CE, Spiro A, Baldwin C, Whelan K, Thomas JE, et al. ( 2009) A review of the evidence for the impact of improving nutritional care on nutritional and clinical outcomes and cost. J Hum Nutr Diet 22 (4): 324-335.

8. Butterworth CE Jr. (1974) The skeleton in the hospital closet. Nutrition 10(5): 442. 